\title{
NUMERICAL VERIFICATION METHOD FOR SOLUTIONS OF THE PERTURBED GELFAND EQUATION*
}

\author{
TERUYA MINAMOTO ${ }^{\dagger}$, NOBITO YAMAMOTO $\ddagger$, AND MITSUHIRO T. NAKAO $\$$
}

\begin{abstract}
A numerical verification method for radially symmetric solutions of the perturbed Gelfand equation is presented for the case in which this equation possesses turning points. We use Nakao's method with local uniqueness to enclose the continua of solutions and a bordering algorithm in order to treat a turning point. We describe verification procedures in detail and give a numerical example.
\end{abstract}

1. Introduction. We consider radially symmetric solutions of the perturbed Gelfand equation

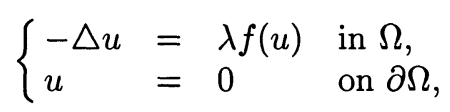

where $\Omega=\left\{x \in R^{n}|| x \mid<1\right\}(n \geq 3), f(u)=\exp (u /(1+\varepsilon u)), \lambda \in R$, and $\varepsilon \in R^{+}$. This equation arises in the theory of combustion and was proposed by D.A. FrankKamenetskii [4]. For some fixed $\varepsilon$, the bifurcation diagram possesses turning points, the first of which corresponding to an explosive point. This explosive point is often denoted by $\lambda_{F K}[2,4]$. The equation (1.1) has been discussed by several authors $[3,9,10,15,16]$. For $\varepsilon=0$ a numerically verified value of $\lambda_{F K}$ was obtained on the unit square[10]. However, the value for $\varepsilon>0$ has not yet been done.

In this paper, we propose a numerical verification method for the existence and enclosure of solution curves for (1.1). If this method can be made to succeed near the first turning point, we can obtain a value for $\lambda_{F K}$. Briefly stated, our method consists of a combination of Nakao's method, (more precisely, it's extension to local uniqueness[19]) with linear interpolation and the implicit function theorem. By adjoining to (1.1) a suitably chosen equation characterized by a new independent parameter $\mu$, we can produce an equation which possesses no turning points, at least locally. This is carried out by applying a "bordering algorithm" [6]. We combine the bordering algorithm with the existence and inclusion method mentioned above to obtain the desired results near turning points.

2. Change of parameters. A radially symmetric solution $u$ of (1.1) is a function of $r=|x|$. Assuming $u$ to be such a function, (1.1) is reduced to the ordinary differential equation

$$
\left\{\begin{array}{l}
-u_{r r}-\frac{n-1}{r} u_{r}-\lambda f(u)=0 \text { in } J \equiv(0,1), \\
u_{r}(0)=u(1)=0 .
\end{array}\right.
$$

Moreover, (2.1) can be transformed into the following integral equation:

$$
u(r)=\frac{\lambda}{n-2} \int_{r}^{1}\left(1-s^{n-2}\right) s f(u) d s+\frac{\lambda}{n-2} \int_{0}^{r}\left(\frac{1}{r^{n-2}}-1\right) s^{n-1} f(u) d s .
$$

\footnotetext{
*Received March 23, 1998; revised June 9, 1999.

†Department of Information Science, Saga University, Saga 840-8502, Japan (minamoto@ma. is.saga-u.ac.jp).

$\ddagger$ Department of Computer Science and Information Mathematics, The University of ElectroCommunications, Tokyo 182-8585, Japan (yamamoto@im.uec.ac.jp).

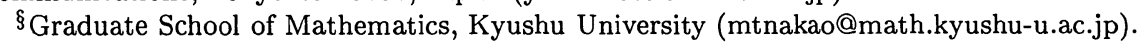


Now, we define the operators $L_{1}: C[0,1] \times R \rightarrow C[0,1]$ and $F_{1}: C[0,1] \times R \rightarrow$ $C[0,1]$ by

$$
L_{1}(u, \lambda)=u
$$

and

$$
F_{1}(u, \lambda)=\frac{\lambda}{n-2} \int_{r}^{1}\left(1-s^{n-2}\right) s f(u) d s+\frac{\lambda}{n-2} \int_{0}^{r}\left(\frac{1}{r^{n-2}}-1\right) s^{n-1} f(u) d s
$$

Then (2.2) can be written as

$$
L_{1}(u, \lambda)=F_{1}(u, \lambda) .
$$

Next, we define the subsets $\mathrm{R}\left(L_{1}-F_{1}\right) \subset C[0,1] \times R$ and $\mathrm{M}_{0} \subset \mathrm{R}\left(L_{1}-F_{1}\right)$ by

$$
\mathrm{R}\left(L_{1}-F_{1}\right) \equiv\left\{(u, \lambda) \in C[0,1] \times R \mid D\left(L_{1}-F_{1}\right)(u, \lambda) \text { is onto }\right\}
$$

and

$$
\mathrm{M}_{0} \equiv\left\{(u, \lambda) \in \mathrm{R}\left(L_{1}-F_{1}\right) \mid\left(L_{1}-F_{1}\right)(u, \lambda)=0\right\},
$$

where $D\left(L_{1}-F_{1}\right)(u, \lambda)$ represents the Fréchet derivative of $\left(L_{1}-F_{1}\right)$ at $(u, \lambda)$. By the Fink-Rheinboldt theory(Theorem 4.1, $4.2[11]), \mathrm{R}\left(L_{1}-F_{1}\right)$ is open and $\mathrm{M}_{0}$ is an one-dimensional manifold, because $L_{1}-F_{1}$ is a Fredholm mapping with index 1. Moreover, we can conclude that $\operatorname{Ker} D\left(L_{1}-F_{1}\right)(u, \lambda)$ is one-dimensional for any $(u, \lambda) \in \mathrm{R}\left(L_{1}-F_{1}\right)$ by using the same argument in [17]. by

Consider some fixed $\mu \in R$ and $r_{i_{c}} \in J$. We define $G_{1}: \mathrm{R}\left(L_{1}-F_{1}\right) \rightarrow C[0,1] \times R$

$$
G_{1}(u, \lambda) \equiv\left(\left(L_{1}-F_{1}\right)(u, \lambda), u\left(r_{i_{c}}\right)-\mu\right)
$$

for $(u, \lambda) \in \mathrm{R}\left(L_{1}-F_{1}\right)$. Then we have

$$
D G_{1}(u, \lambda)(\psi, \gamma)=\left(D_{u}\left(L_{1}-F_{1}\right)(u, \lambda) \psi+\gamma D_{\lambda}\left(L_{1}-F_{1}\right)(u, \lambda), \psi\left(r_{i_{c}}\right)\right)
$$

for $\gamma \in R$ and $\psi \in C[0,1]$, where $D_{u}$ and $D_{\lambda}$ denote partial derivatives with respect to $u$ and $\lambda$, respectively.

We need a result similar to that obtained in [17]. The proof of the following lemma can be carried out by the argument appearing there.

LEMMA 1. Let $(u, \lambda) \in \mathrm{R}\left(L_{1}-F_{1}\right)$ and let $\left(\psi_{0}, \gamma_{0}\right)$ be a basis of $\operatorname{KerD}\left(L_{1}-\right.$ $\left.F_{1}\right)(u, \lambda)$. Then with $r_{i_{c}} \in J$ such that $\psi_{0}\left(r_{i_{c}}\right) \neq 0$,

$$
D G_{1}(u, \lambda): C[0,1] \times R \rightarrow C[0,1] \times R
$$

is bijection.

To make a change of parameters, we choose $r_{i_{c}}$ as required in Lemma 1.

From the implicit function theorem $([20])$, for any $(u, \lambda) \in M_{0}$, there exist an $\varepsilon_{0}>0$ and a unique Fréchet differentiable map

$$
\left(u\left(r_{i_{c}}\right)-\varepsilon_{0}, u\left(r_{i_{c}}\right)+\varepsilon_{0}\right) \ni \mu \mapsto(u(\mu), \lambda(\mu)) \in M_{0}
$$

such that $(u, \lambda)=\left(u\left(\mu_{0}\right), \lambda\left(\mu_{0}\right)\right)$ with $\mu_{0} \equiv u\left(r_{i_{c}}\right)$. 
Also, for any $\mu \in\left(u\left(r_{i_{c}}\right)-\varepsilon_{0}, u\left(r_{i_{c}}\right)+\varepsilon_{0}\right)$, the relation $G_{1}(u(\mu), \lambda(\mu))=(0,0)$ holds. That is,

$$
\left(L_{1}-F_{1}\right)(u(\mu), \lambda(\mu))=0 \text { and } u(\mu)\left(r_{i_{c}}\right)=\mu \text {. }
$$

Below, we present a method for choosing $r_{i_{c}} \in J$. For this purpose, we give the following lemma.

Lemma 2. [11] For any $(u, \lambda) \in \mathrm{M}_{0}$, the tangent space $T_{(u, \lambda)} \mathrm{M}_{0}$ is identical to the null-space $\operatorname{KerD}\left(L_{1}-F_{1}\right)(u, \lambda)$.

Now, let $\Delta: 0=r_{0}<r_{1}<\cdots<r_{M-1}<r_{M}=1$ be a uniform partition of $J$ into subintervals $\left[r_{j}, r_{j+1}\right]$ of length $h=r_{j+1}-r_{j}(j=0, \cdots, M-1)$, and let $S_{h} \subset C[0,1]$ be a finite dimensional space depending on $h$ which has the hat functions $\phi_{j}(j=0,1, \cdots, M-1)$ as a basis.

Then, we consider the following approximations of $\mathrm{R}\left(L_{1}-F_{1}\right)$ and $\mathrm{M}_{0}$ :

$$
\mathrm{R}_{h}\left(L_{1}-F_{1}\right)=\left\{\left(u_{h}, \lambda_{h}\right) \in S_{h} \times R \mid D \Pi_{h 0}\left(L_{1}-F_{1}\right)\left(u_{h}, \lambda_{h}\right) \text { is onto }\right\}
$$

and

$$
\mathrm{M}_{0 h}=\left\{\left(u_{h}, \lambda_{h}\right) \in \mathrm{R}_{h}\left(L_{1}-F_{1}\right) \mid \Pi_{h 0}\left(L_{1}-F_{1}\right)\left(u_{h}, \lambda_{h}\right)=0\right\} .
$$

Here $\Pi_{h 0}: C[0,1] \rightarrow S_{h}$ is an interpolation operator defined by

$$
\phi\left(r_{j}\right)=\Pi_{h 0} \phi\left(r_{j}\right) \quad j=0,1, \cdots, M-1 .
$$

Since we can show that $M_{0 h}$ is one-dimensional manifold by using the same argument in [Lemma 5.1, [11]], we can consider the tangent space $T_{\left(u_{h}, \lambda_{h}\right)} \mathrm{M}_{0_{h}}$. Based on Lemma 2 , we compute the tangent vector $t_{h} \in T_{\left(u_{h}, \lambda_{h}\right)} \mathrm{M}_{0 h}$ of the manifold $\mathrm{M}_{0 h}$ approximately, and choose the continuation index $i_{c}$ so that $\left|\psi_{0 h}\left(r_{i_{c}}\right)\right|=\left\|t_{h}\right\|_{\infty}$, where $\psi_{0_{h}}$ is the basis of $T_{\left(u_{h}, \lambda_{h}\right)} \mathrm{M}_{0 h}$ and $r_{i_{c}}$ is a nodal point of $S_{h}$. Then, choosing $h>0$ sufficiently small, $\left|\psi_{0 h}\left(r_{i_{c}}\right)\right|$ can be made arbitrarily close to $\left\|\psi_{0}\left(r_{i_{c}}\right)\right\|$, and $\left\|\psi_{0}\left(r_{i_{c}}\right)\right\| \neq 0$. Therefore , we may expect $D G_{1}(u, \lambda)$ is bijection for such $r_{i_{c}}$ by Lemma 1 .

3. Enclosure of solutions with local uniqueness for a fixed parameter $\mu$. The arguments outlined in this section are very similar to those in [19]. We include this outline to make the present paper self-contained.

We define the operator $\Pi_{h}: C[0,1] \times R \rightarrow S_{h} \times R$ by

$$
\Pi_{h}(u, \lambda) \equiv\left(\Pi_{h 0} u, \lambda\right) \quad \text { for }(u, \lambda) \in C[0,1] \times R .
$$

Let $\left(u_{h}, \lambda_{h}\right) \in S_{h} \times R$ be an approximate solution of (2.8). We introduce the following "residual" fixed point form which corresponds to (2.8):

$$
\begin{aligned}
(\widetilde{u}, \tilde{\lambda})= & \left(F_{1}\left(u_{h}+\widetilde{u}, \lambda_{h}+\tilde{\lambda}\right)-u_{h}, \tilde{\lambda}-\left(u_{h}+\widetilde{u}\right)\left(r_{i_{c}}\right)+\mu\right) \\
= & \left(\Pi_{h 0} F_{1}\left(u_{h}+\widetilde{u}, \lambda_{h}+\tilde{\lambda}\right)-u_{h}\right. \\
& \left.\quad+\left(I-\Pi_{h 0}\right) F_{1}\left(u_{h}+\tilde{u}, \lambda_{h}+\widetilde{\lambda}\right), \tilde{\lambda}-\left(u_{h}+\widetilde{u}\right)\left(r_{i_{c}}\right)+\mu\right) \\
\equiv & F(\widetilde{u}, \widetilde{\lambda}) \quad(\widetilde{u}, \tilde{\lambda}) \in C[0,1] \times R .
\end{aligned}
$$

This equation can also be written as

$$
\left\{\begin{array}{lll}
\Pi_{h}(\widetilde{w}) & =\Pi_{h} F(\widetilde{w}) \\
\left(I-\Pi_{h}\right)(\widetilde{w}) & =\left(I-\Pi_{h}\right) F(\widetilde{w}),
\end{array}\right.
$$


where $\widetilde{w}=(\widetilde{u}, \widetilde{\lambda})$, and $I$ represents the identity map on $C[0,1] \times R$.

Assumption 1. Suppose that restriction to $S_{h} \times R$ of the operator $\Pi_{h}[I-D F(0)]$ : $C[0,1] \times R \rightarrow S_{h} \times R$ has the inverse

$$
\left[I_{h}-A_{h}^{\prime}\right]^{-1}: S_{h} \times R \rightarrow S_{h} \times R,
$$

where $I_{h}=\Pi_{h} I$ and $A_{h}^{\prime}$ is a linear operator on $S_{h} \times R$, an approximation to $\Pi_{h} D F(0)$.

We apply a Newton-like method to the first equation in (3.2). That is, we introduce the operator as follows:

$$
\Pi_{h} N \equiv \Pi_{h}-\left[I_{h}-A_{h}^{\prime}\right]^{-1} \Pi_{h}(I-F) .
$$

Then we obtain

$$
\widetilde{w}=T(\widetilde{w}) .
$$

Here $T$ is the operator on $C[0,1] \times R$ defined by $T \equiv \Pi_{h} N+\left(I-\Pi_{h}\right) F$. It is easy to see that $\widetilde{w}=T(\widetilde{w})$ and $\widetilde{w}=F(\widetilde{w})$ are equivalent.

We now expand the operator $T$ at 0 and describe the verification conditions with local uniqueness by using Banach's fixed point theorem. Setting $w=(u, \lambda)=$ $\left(\Pi_{h 0} u+\left(I-\Pi_{h 0}\right) u, \lambda\right)$, we write $\Pi_{h 0} u=\sum_{j=0}^{M-1} u_{i} \phi_{i}$ and define

$$
\begin{aligned}
(w)_{i} & \equiv\left|u_{i}\right| \quad(i=0,1, \cdots, M-1) \\
(w)_{M} & \equiv|\lambda|, \\
(w)_{M+1} & \equiv\left\|\left(I-\Pi_{h 0}\right) u\right\|_{C[0,1]},
\end{aligned}
$$

where $\|v\|_{C[0,1]} \equiv \max _{r \in J}|v(r)|$, for $v \in C[0,1]$.

Choosing a positive vector $\vec{W}=\left(\widetilde{W}_{0}, \widetilde{W}_{1}, \cdots, \widetilde{W}_{M}, \widetilde{W}_{M+1}\right)^{t} \in R^{M+2}$, we define the set $W$ by

$$
W \equiv\left\{\widetilde{w} \in C[0,1] \times R \mid(\widetilde{w})_{i} \leq \widetilde{W}_{i}, i=0,1, \cdots, M+1\right\} .
$$

Next, we choose the vectors

$$
\begin{aligned}
& \left(\tilde{Y}_{0}, \cdots, \widetilde{Y}_{M+1}\right)^{t} \in R^{M+2}, \tilde{Y}_{i}>0(i=0, \cdots, M+1), \\
& \left(\widetilde{Z}_{0}, \cdots, \widetilde{Z}_{M+1}\right)^{t} \in R^{M+2}, \widetilde{Z}_{i}>0(i=0, \cdots, M+1)
\end{aligned}
$$

such that

$$
\begin{array}{rlrl}
(T(0))_{i} & \leq \widetilde{Y}_{i} & & (i=0, \cdots, M+1), \\
\left(D T\left(\widetilde{w_{1}}\right) \widetilde{w_{2}}\right)_{i} & \leq \widetilde{Z}_{i} \quad(i=0, \cdots, M+1) \quad \forall \widetilde{w_{1}}, \widetilde{w_{2}} \in W,
\end{array}
$$

and we define the set $\widetilde{K}$ in $C[0,1] \times R$ by

$$
\widetilde{K} \equiv\left\{v \in C[0,1] \times R \mid(v)_{i} \leq \tilde{Y}_{i}+\widetilde{Z}_{i}, i=0, \cdots, M+1\right\} .
$$

Then the verification condition is described as follows: 
TheOREM 1 (Local Uniqueness). If $\widetilde{K} \subset W$ holds for $W$ (that is, if $\widetilde{Y}_{i}+\widetilde{Z}_{i} \leq$ $\left.\widetilde{W}_{i}\right)$, then there exists a solution to

$$
\widetilde{w}=T(\widetilde{w})
$$

in $\widetilde{K}$ and it is unique within the set $W$.

\section{Outline of proof:}

First, we define a scaling norm $\|\cdot\|_{W}$ by

$$
\|w\|_{\widetilde{W}} \equiv \max _{0 \leq i \leq M+1} \frac{(\widetilde{w})_{i}}{\widetilde{W}_{i}} \quad \forall w \in C[0,1] \times R .
$$

Second, we obtain the following two relations from the definition of this norm:

$$
T(W) \subset W \text { and }\left\|T\left(w_{2}\right)-T\left(w_{1}\right)\right\|_{W} \leq k\left\|w_{2}-w_{1}\right\|_{W} \quad \forall w_{1}, w_{2} \in W
$$

for some $k$ satisfying $0<k<1$. Then Banach's fixed point theorem gives the desired result.

4. Enclosing continua of solutions $\left(u^{(\mu)}, \lambda^{(\mu)}\right)_{\mu \in e_{0}}$ for a small interval $e_{0}$.

4.1. Verification condition. We proceed to extend the results of Section 3 for continua of solutions $\widetilde{w}_{(\mu)}:=\left(\widetilde{u}^{(\mu)}, \widetilde{\lambda}^{(\mu)}\right)_{\mu \in e_{0}}$ depending smoothly on $\mu$. Here $e_{0}$ denotes a small real interval, and we set $e_{0}=\left[\mu_{0}, \mu_{1}\right]$.

Let $\left(u_{h}^{0}, \lambda_{h}^{0}\right)$ and $\left(u_{h}^{1}, \lambda_{h}^{1}\right)$ be the approximate solutions of (2.8) corresponding to $\mu_{0}$ and $\mu_{1}$, respectively. We define approximate solutions for all $\mu \in e_{0}$ as follows:

$$
u_{h}^{(\mu)}=\frac{\mu_{1}-\mu}{\mu_{1}-\mu_{0}} u_{h}^{0}+\frac{\mu-\mu_{0}}{\mu_{1}-\mu_{0}} u_{h}^{1}, \quad \lambda_{h}^{(\mu)}=\frac{\mu_{1}-\mu}{\mu_{1}-\mu_{0}} \lambda_{h}^{0}+\frac{\mu-\mu_{0}}{\mu_{1}-\mu_{0}} \lambda_{h}^{1} .
$$

Then we define the mappings $F$ and $T$ for $\left(\mu, u_{h}^{(\mu)}, \lambda_{h}^{(\mu)}\right) \in e_{0} \times S_{h} \times R$ as in Section 3 , and denote them as $F_{(\mu)}$ and $T_{(\mu)}$. That is, for $\widetilde{w}=(\widetilde{u}, \widetilde{\lambda}) \in C[0,1] \times R$,

$$
\begin{aligned}
F_{(\mu)}(\widetilde{u}, \widetilde{\lambda}) & =\left(F_{1}\left(u_{h}^{(\mu)}+\widetilde{u}, \lambda_{h}^{(\mu)}+\widetilde{\lambda}\right)-u_{h}^{(\mu)}, \tilde{\lambda}-\left(u_{h}^{(\mu)}+\widetilde{u}\right)\left(r_{i_{c}}\right)+\mu\right), \\
T_{(\mu)}(\widetilde{w}) & =\Pi_{h} N_{(\mu)}(\widetilde{w})+\left(I-\Pi_{h}\right) F_{(\mu)}(\widetilde{w}),
\end{aligned}
$$

where $\Pi_{h} N_{(\mu)}(\widetilde{w})=\Pi_{h} \widetilde{w}-\left[I_{h}-A_{h, \bar{\mu}}^{\prime}\right]^{-1} \Pi_{h}\left(I-F_{(\mu)}\right)(\widetilde{w})$ and $A_{h, \bar{\mu}}^{\prime}$ denotes a linear operator on $S_{h} \times R$, an approximation of $\Pi_{h} D F_{(\bar{\mu})}(0)$ for some fixed $\bar{\mu} \in e_{0}$.

Choosing the vectors $\vec{Y}=\left(Y_{0}, \cdots, Y_{M+1}\right)^{t} \in R^{M+2}$ and $\vec{Z}=\left(Z_{0}, \cdots, Z_{M+1}\right)^{t} \in$ $R^{M+2}$ such that

$$
\max _{\mu \in e_{0}}\left(T_{(\mu)}(0)\right)_{i} \leq Y_{i}
$$

and

$$
\max _{\mu \in e_{0}}\left(D T_{(\mu)}\left(\widetilde{w}_{1}\right) \widetilde{w}_{2}\right)_{i} \leq Z_{i}, \quad \forall \widetilde{w}_{1}, \widetilde{w}_{2} \in W
$$

and defining the set $K$ by

$$
K \equiv\left\{v \in C[0,1] \times R \mid(v)_{i} \leq Y_{i}+Z_{i}, i=0,1, \cdots, M+1\right\},
$$

the following theorem holds for the set $W$ which is defined by (3.6).

THEOREM 2 (Uniqueness for small interval). If $K \subset W$, then there exists a solution to

$$
\widetilde{w}_{(\mu)}=T_{(\mu)}\left(\widetilde{w}_{(\mu)}\right)
$$

in $K$ for all $\mu \in e_{0}$, and it is unique within the set $W$. 
4.2. Verification Procedures by Computer. Defining the set $W$, we estimate $K$ in Theorem 2 by computer. For the set $K$, we must estimate $T_{(\mu)}(0)$ and $D T_{(\mu)}\left(\widetilde{w}_{1}\right) \widetilde{w}_{2}$ for any $\widetilde{w}_{1}, \widetilde{w}_{2} \in W$. First we note

$$
T_{(\mu)}(0)=\left[I_{h}-A_{h, \bar{\mu}}^{\prime}\right]^{-1} \Pi_{h} F_{(\mu)}(0)+\left(I-\Pi_{h}\right) F_{(\mu)}(0) .
$$

We denote the finite part and the infinite one of $T_{(\mu)}(0)$ by $T_{h}^{(\mu)}(0)$ and $T_{\perp}^{(\mu)}(0)$, respectively, and consider a vector $\overrightarrow{g h} \in R^{M+1}$ whose elements are given by $\left[g_{h}\right]_{i}$ :

$$
\begin{aligned}
{\left[g_{h}\right]_{i} } & =\left\{\Pi_{h 0} F_{1}\left(u_{h}^{(\mu)}, \lambda_{h}^{(\mu)}\right)\left(r_{i}\right)-u_{h}^{(\mu)}\left(r_{i}\right) \mid \mu \in e_{0}\right\} \quad i=0,1, \cdots, M-1, \\
{\left[g_{h}\right]_{M} } & =\left\{\mu-u_{h}^{(\mu)}\left(r_{i_{c}}\right) \mid \mu \in e_{0}\right\} .
\end{aligned}
$$

In order to compute a vector $\overrightarrow{T_{h}^{(\mu)}}(0)$ which consists of elements $\max _{\mu \in e_{0}}\left(T_{h}^{(\mu)}(0)\right)_{i}$, $i=0,1, \cdots, M$, we solve the following equation

$$
G_{h} \overrightarrow{T_{h}^{(\mu)}}(0)=\overrightarrow{g h}
$$

Here $G_{h}$ is an $(M+1) \times(M+1)$ matrix which corresponds to $\left[I_{h}-A_{h, \bar{\mu}}^{\prime}\right]$. We estimate $\overrightarrow{g h}$ by interval value, because it is difficult to obtain an exact value of $\left[g_{h}\right]_{i}$. Using this interval solution of (4.2), we can determine $Y_{i}$ satisfying

$$
\max _{\mu \in e_{0}}\left(T_{h}^{(\mu)}(0)\right)_{i} \leq Y_{i}, \quad i=0,1, \cdots, M
$$

To obtain an upper bound for $T_{\perp}^{(\mu)}(0)$, we use an error estimation for the linear interpolation:

Proposition 1 (Error estimation for linear interpolation [14]) The relation

$$
\left\|\left(I-\Pi_{h 0}\right) v\right\|_{\infty} \leq \frac{h^{2}}{8}\left\|\frac{d^{2} v}{d r^{2}}\right\|_{\infty}
$$

holds for all $v \in C[0,1] \cup C^{2, \infty}(0,1)$, where $C^{2, \infty}(0,1):=\left\{v \in C^{2}(0,1)\|\| \frac{d^{2} v}{d r^{2}} \|_{\infty}<\right.$ $\infty\}$.

From the above proposition we have

$$
\max _{\mu \in e_{0}}\left\|T_{\perp}^{(\mu)}(0)\right\|_{C[0,1] \times R} \leq \frac{h^{2}}{8} \max _{\mu \in e_{0}}\left\|\frac{d^{2}}{d r^{2}} F_{1}\left(u_{h}^{(\mu)}, \lambda_{h}^{(\mu)}\right)\right\|_{C[0,1]}=: Y_{M+1} .
$$

On the other hand, the operator $D T_{(\mu)}\left(\widetilde{w}_{1}\right)$ is defined by

$$
\begin{aligned}
D T_{(\mu)}\left(\widetilde{w}_{1}\right) \widetilde{w}_{2} & =\left[I_{h}-A_{h, \bar{\mu}}^{\prime}\right]^{-1} \Pi_{h}\left(D F_{(\mu)}\left(\widetilde{w}_{1}\right) \widetilde{w}_{2}-A_{h, \bar{\mu}}^{\prime} \Pi_{h} \widetilde{w}_{2}\right) \\
& +\left(I-\Pi_{h}\right) D F_{(\mu)}\left(\widetilde{w}_{1}\right) \widetilde{w}_{2} \quad \widetilde{w}_{1}, \widetilde{w}_{2} \in W .
\end{aligned}
$$

Using $(\widetilde{w})_{i} \leq \widetilde{W}_{i}(i=0,1, \cdots, M+1)$ for $\tilde{w} \in W$, we can estimate $\max _{\mu \in e_{0}}\left(D T_{(\mu)}\left(\widetilde{w}_{1}\right) \widetilde{w}_{2}\right)_{i}$ by solving some interval linear equations in the similar manner as in the case of $Y_{i}$ (See [19] for detail).

We now describe how to obtain a set $W$ which is specified by $\vec{W}$. 
First, we take the initial value $\widetilde{W}_{i, 0}$ of $\widetilde{W}_{i}$ as

$$
\widetilde{W}_{i, 0}=Y_{i} \quad i=0,1, \cdots, M+1,
$$

and apply the following procedure. Considering that $D T_{(\mu)}\left(\widetilde{w}_{1}\right) \widetilde{w}_{2}$ depends on $\vec{W}$, $\left(\max _{\mu \in e_{0}} D T_{(\mu)}\left(\widetilde{w}_{1}\right) \widetilde{w}_{2}\right)_{i}$ is denoted by $Z_{i}(\vec{W}), i=0,1, \cdots, M+1$ for any $\widetilde{w}_{1}, \widetilde{w}_{2} \in W$.

(i) Check the conditions

$$
Y_{i}+Z_{i}\left(\vec{W}_{k}\right) \leq \widetilde{W}_{i, k} \quad(i=0,1, \cdots, M+1),
$$

where $k$ stands for the iteration number. If the above conditions are satisfied, then stop. This means that verification is completed.

(ii) Otherwise, take

$$
\widetilde{W}_{i, k+1}=(1+\delta)\left(Y_{i}+Z_{i}\left(\vec{W}_{k}\right)\right) \quad(i=0,1, \cdots, M+1),
$$

for a certain positive number $\delta$ and return to (i) after re-computing $Z_{i}$.

4.3. Smoothness for a continuum of solutions. We define the mapping $G: \operatorname{int}\left(e_{0}\right) \times C[0,1] \times R \rightarrow C[0,1] \times R$ as

$$
G(\mu, \tilde{u}, \tilde{\lambda}):=\left(I-F_{(\mu)}\right)(\widetilde{u}, \tilde{\lambda}),
$$

and consider the following equation with respect to $\left(\psi_{0}, \gamma_{0}\right)$ :

$$
\begin{aligned}
D_{(\tilde{u}, \widetilde{\lambda})} G\left(\mu, \widetilde{w}_{(\mu)}\right)\left(\psi_{0}, \gamma_{0}\right) & =\left(D_{(\widetilde{u}, \widetilde{\lambda})}\left(L_{1}-F_{1}\right)\left(u_{h}^{(\mu)}+\widetilde{u}^{(\mu)}, \lambda_{h}^{(\mu)}+\widetilde{\lambda}^{(\mu)}\right)\left(\psi_{0}, \gamma_{0}\right), \psi_{0}\left(r_{i_{c}}\right)\right) \\
(4.4) & =(0,1) .
\end{aligned}
$$

Next we apply the verification method presented in the previous section to (4.4). That is, first, transforming (4.4) into the fixed-point form and using a Newton-Like method, (4.4) can be written as $\left(\psi_{0}, \gamma_{0}\right)=\widehat{T}_{\left(\mu, \widetilde{w}_{(\mu)}\right)}\left(\psi_{0}, \gamma_{0}\right)$, where $\widehat{T}$ is defined in an analogous way as $T$ in $\S 3$. Second, for this equation we choose a set $\Phi$ like as $W$ in $\S 3$. Considering the vectors $\widehat{Y}$ and $\widehat{Z} \in R^{M+2}$ such that $\max _{\mu \in e_{0}} \max _{\widetilde{w}_{(\mu)} \in W}\left(\widehat{T}_{\left(\mu, \widetilde{w}_{(\mu)}\right)}(0)\right)_{i} \leq \widehat{Y}_{i}$ and $\max _{\mu \in e_{0}} \max _{\widetilde{w}_{(\mu)} \in W}\left(D \widehat{T}_{\left(\mu, \tilde{w}_{(\mu)}\right)}\left(\phi_{1}\right) \phi_{2}\right)_{i} \leq \widehat{Z}_{i}, \phi_{1}, \phi_{2} \in \Phi$, we define $\widehat{K}$ by

$$
\widehat{K}:=\left\{v \in C[0,1] \times R \mid(v)_{i} \leq \widehat{Y}_{i}+\widehat{Z}_{i}, i=0,1, \cdots, M+1\right\} .
$$

Then we have the following:

LemMA 3. If $\widehat{K} \subset \Phi$ holds for $\Phi$, then the mapping $D G_{(\widetilde{u}, \widetilde{\lambda})}\left(\mu, \widetilde{w}_{(\mu)}\right)$ is a bijection for each $\mu \in e_{0}$.

Proof. If the assumption holds, there exists a unique solution to (4.4) in $\widehat{K}$ for all $\mu \in e_{0}$. From this fact, it follows that the equation

$$
D_{(\widetilde{u}, \tilde{\lambda})} G\left(\mu, \widetilde{w}_{(\mu)}\right)\left(\psi_{1}, \gamma_{1}\right)=(0,0)
$$

has no nontrivial solution. Since the linear mapping $D_{(\widetilde{u}, \widetilde{\lambda})} G\left(\mu, \widetilde{w}_{(\mu)}\right)$ is a Fredholm operator with index 0 from the compactness of $D_{(\widetilde{u}, \tilde{\lambda})} F_{(\mu)}(\widetilde{u}, \widetilde{\lambda}), D_{(\widetilde{u}, \widetilde{\lambda})} G\left(\mu, \widetilde{w}_{(\mu)}\right)$ is onto according to the Fredholm alternative([5]). 
The following theorem is a modified version of a similar result obtained in [10].

THEOREM 3 (The smoothness of solutions). If $K \stackrel{\circ}{\subset} W$ and $\widehat{K} \subset \Phi$, then there exists a Fréchet differentiable mapping:

$$
\operatorname{int}\left(e_{0}\right) \ni \mu \longmapsto \widetilde{w}_{(\mu)} \in W
$$

such that $G\left(\mu, \widetilde{w}_{(\mu)}\right)=0$ on $\mu \in \operatorname{int}\left(e_{0}\right)$.

Proof. From Lemma 3, for some arbitrarily chosen $\left(\bar{\mu}, \widetilde{w}_{(\bar{\mu})}\right) \in \operatorname{int}\left(e_{0}\right) \times W$, the implicit function theorem gives an open interval $V \subset \operatorname{int}\left(e_{0}\right)$ containing $\bar{\mu}$, and a Fréchet differentiable mapping

$$
V \ni \mu \longmapsto \widehat{w}_{(\mu)} \in C[0,1] \times R
$$

such that $G\left(\mu, \widehat{w}_{(\mu)}\right)=0$ for $\mu \in V$ and $\widehat{w}_{(\bar{\mu})}=\widetilde{w}_{(\bar{\mu})}$. Without loss of generality, we can assume $V$ is maximal with property (4.5).

Let $\widehat{V}$ denote the maximal subinterval of $V$ containing $\bar{\mu}$ such that

$$
\widehat{w}_{(\mu)} \in W \quad \text { for all } \mu \in \widehat{V}
$$

that is, we take a mapping : $\widehat{V} \ni \mu \mapsto \widehat{w}_{(\mu)} \in W$. If we prove $\widehat{V}=\operatorname{int}\left(e_{0}\right)$, then we can conclude that $\widehat{w}_{(\mu)}=\widetilde{w}_{(\mu)}$ for all $\mu \in \operatorname{int}\left(e_{0}\right)$ due to the uniqueness of solutions in $W$.

Assuming that $\widehat{V} \neq \operatorname{int}\left(e_{0}\right)$, there exists some $\mu^{*} \in \operatorname{int}\left(e_{0}\right) \cap \partial \widehat{V}$. Applying the implicit function theorem at $\left(\mu^{*}, \widetilde{w}_{\left(\mu^{*}\right)}\right)$, we can obtain an open interval $U \subset \operatorname{int}\left(e_{0}\right)$ containing $\mu^{*}$ and a Fréchet differentiable mapping,

$$
U \ni \mu \longmapsto \bar{w}_{(\mu)} \in C[0,1] \times R,
$$

such that $G\left(\mu, \bar{w}_{(\mu)}\right)=0$ for $\mu \in U$ and $\bar{w}_{\left(\mu^{*}\right)}=\widetilde{w}_{\left(\mu^{*}\right)}$.

From the fact that $\bar{w}_{\left(\mu^{*}\right)} \in T_{\left(\mu^{*}\right)} W \stackrel{\circ}{\subset} W$, the relation $\bar{w}_{\left(\mu^{*}\right)} \in \operatorname{int}(W)$ holds.

Thus, for some neighborhood $\widetilde{U}$ of $\mu^{*}$, the continuity of $\bar{w}_{(\mu)}$ with respect to $\mu \in U$ gives the relation

$$
\bar{w}_{(\mu)} \in W \quad \text { for all } \mu \in \tilde{U} .
$$

From (4.6), (4.8) and the uniqueness of solution in $W$, we obtain

$$
\widehat{w}_{(\mu)}=\bar{w}_{(\mu)} \quad \text { for all } \mu \in \widehat{V} \cap \widetilde{U} .
$$

Then, since $\tilde{U}$ is an open interval containing $\mu^{*} \in \partial \widehat{V}$, it follows that $\widehat{V} \underset{\neq}{\subset} \cup \widetilde{U}$.

By (4.9), $\widehat{w}_{(\mu)}$ can be extended to the open interval $\widehat{V} \cup \widetilde{U}$ by $\bar{w}_{(\mu)}$, while $\widehat{V} \cup \widetilde{U} \subset V$ holds due to the maximality of $V$. This implies the relations $\widehat{V} \underset{\neq}{ } \widehat{V} \cup \widetilde{U} \subset V$, but this contradicts the maximality of $\widehat{V}$.

5. Verification for a large interval of parameters. In this section, we assume that the verification process on each small interval $e_{i}=\left[\mu_{i}, \mu_{i+1}\right](i=$ $0,1,2, \cdots, N)$ has succeeded for some fixed natural number $N$, that is, the assumptions of Theorem 3 are required for each $i=0,1,2, \cdots, N$. 
THEOREM 4. Let $W^{(i)}$ be an enclosure set of solutions for parameters $\mu \in e_{i}$. If

$$
K^{(i)} \subset W^{(i+1)}
$$

or

$$
K^{(i+1)} \subset W^{(i)}
$$

then there exists a smooth solution continuum in $W^{(i)} \cup W^{(i+1)}$. Here, $K^{(i)}$ is a set in which there exist solutions for parameters $\mu \in e_{i}$ (see Fig.1).

Proof. Let $K^{(i)} \subset W^{(i+1)}$, and denote the solutions for $e_{i}$ and $e_{i+1}$ as $\left\{\widetilde{w}_{(\mu), i}\right\}_{\mu \in e_{i}}$ $\subset K^{(i)}$ and $\left\{\widetilde{w}_{(\mu), i+1}\right\}_{\mu \in e_{i+1}} \subset W^{(i+1)}$, respectively. By the fact that verification has succeed for $e_{i}$ and $e_{i+1}$, there exist unique $\left\{\widetilde{w}_{\left(\mu_{i+1}\right), i}\right\} \in K^{(i)}$ and $\left\{\widetilde{w}_{\left(\mu_{i+1}\right), i+1}\right\} \in$ $W^{(i+1)}$ which are solutions on the contact point $\mu_{i+1}$ between $e_{i}$ and $e_{i+1}$. Then, from the assumption $K^{(i)} \subset W^{(i+1)}$ and the local uniqueness,

$$
\widetilde{w}_{\left(\mu_{i+1}\right), i}=\widetilde{w}_{\left(\mu_{i+1}\right), i+1} \in K^{(i)} \subset W^{(i+1)}
$$

holds. This implies that $\left\{\widetilde{w}_{(\mu), i}\right\}_{\mu \in e_{i}} \cup\left\{\widetilde{w}_{(\mu), i+1}\right\}_{\mu \in e_{i+1}}$ is continuous at $\mu_{i+1}$. The remaining problem is to prove that $\left\{\widetilde{w}_{(\mu), i}\right\}_{\mu \in e_{i}} \cup\left\{\widetilde{w}_{(\mu), i+1}\right\}_{\mu \in e_{i+1}}$ is smooth at $\mu_{i+1}$.

From Lemma 3 , the implicit function theorem at $\left(\mu_{i+1}, \widetilde{w}_{\left(\mu_{i+1}, i\right.}\right)$ provides an open interval $V \subset \operatorname{int}\left(e_{i} \cup e_{i+1}\right)$ containing $\mu_{i+1}$ and a Fréchet differentiable mapping,

$$
V \ni \mu \longmapsto \widetilde{w}_{(\mu)} \in C[0,1] \times R,
$$

such that $\widetilde{w}_{\left(\mu_{i+1}\right)}=\widetilde{w}_{\left(\mu_{i+1}\right), i}$ and $G\left(\mu, \widetilde{w}_{(\mu)}\right)=0$ (i.e. $\left.\widetilde{w}_{(\mu)}=F_{(\mu)}\left(\widetilde{w}_{(\mu)}\right)\right)$. Thus $\widetilde{w}_{\left(\mu_{i+1}\right)} \in K^{(i)} \stackrel{\circ}{\complement} W^{(i)}$ implies $\widetilde{w}_{(\mu)} \in W^{(i)}$ in some left neighborhood of $\mu_{i+1}$, and therefore $\widetilde{w}_{(\mu)}=\widetilde{w}_{(\mu), i}$ in this left neighborhood due to uniqueness in $W^{(i)}$. Moreover, $\widetilde{w}_{\left(\mu_{i+1}\right)}=\widetilde{w}_{\left(\mu_{i+1}\right), i+1} \in K^{(i+1)} \stackrel{\circ}{\complement} W^{(i+1)}$ implies $\widetilde{w}_{(\mu)} \in W^{(i+1)}$ in some right neighborhood of $\mu_{i+1}$, and therefore $\widetilde{w}_{(\mu)}=\widetilde{w}_{(\mu), i+1}$ in this right neighborhood due to uniqueness in $W^{(i+1)}$. This implies smoothness of the curve $\left\{\widetilde{w}_{(\mu), i}\right\}_{\mu \in e_{i}} \cup$ $\left\{\widetilde{w}_{(\mu), i+1}\right\}_{\mu \in e_{i+1}}$ at $\mu_{i+1}$.

This argument can be applied to the case of $K^{(i+1)} \subset W^{(i)}$ in a similar manner. $\square$

Finally, we state a lemma that ensures the enclosure of turning points. In the following lemma, we suppose that the set $W^{(i)}$ satisfies the assumptions of Theorem 4 , and $\varepsilon_{i}=\widetilde{W}_{M}^{(i)}$ which is the Mth component of $\overrightarrow{W^{(i)}}$.

LEMMA 4. (Bound for turning points) Let $\mu_{j}<\mu_{k}<\mu_{l}$ for some $0 \leq j<$ $k<l \leq N$ and $I_{i}=\left[\lambda_{h}\left(\mu_{i}\right)-\varepsilon_{i}, \lambda_{h}\left(\mu_{i}\right)+\varepsilon_{i}\right]$. If $I_{j} \cap I_{k}=\phi, I_{k} \cap I_{l}=\phi$, and $I_{j} \cap \bar{I}_{l} \neq \phi$ hold, then, there exists a turning point in $\left[\mu_{j}, \mu_{l}\right]$ (see Fig.2).

Proof. We assume that there is no turning point for any $\mu^{\prime} \in\left(\mu_{j}, \mu_{l}\right)$. Without loss of generality, we may assume that $\lambda\left(\mu_{j}\right) \leq \lambda\left(\mu_{k}\right) \leq \lambda\left(\mu_{l}\right)$. From the fact that the verification process has succeeded, it follows that $\lambda\left(\mu_{i}\right) \in I_{i}$. Then, because $I_{j} \cap I_{k}=\phi$ and $\lambda\left(\mu_{j}\right) \leq \lambda\left(\mu_{k}\right)$

$$
\lambda_{h}\left(\mu_{j}\right)+\varepsilon_{j}<\lambda_{h}\left(\mu_{k}\right)-\varepsilon_{k} .
$$

Similarly, we can obtain

$$
\lambda_{h}\left(\mu_{k}\right)+\varepsilon_{k}<\lambda_{h}\left(\mu_{l}\right)-\varepsilon_{l} .
$$




\begin{tabular}{lllllllll}
\hline \multicolumn{1}{c}{$\mu$} & $\left\|u_{h}\right\|_{\infty}$ & $\lambda_{h}$ & $K_{h}$ & $W_{h}$ & $K_{M}$ & $\widetilde{W}_{M}$ & $K_{M+1}$ & $\widetilde{W}_{M+1}$ \\
\hline 1.32 & 1.32 & 3.39745 & 0.01633 & 0.01782 & 0.03057 & 0.03268 & $4.749 \mathrm{E}-6$ & $5.022 \mathrm{E}-6$ \\
$\vdots$ & $\vdots$ & $\vdots$ & $\vdots$ & $\vdots$ & $\vdots$ & $\vdots$ & $\vdots$ & $\vdots$ \\
1.818 & 1.818 & 3.5267413 & 0.02106 & 0.02275 & 0.03482 & 0.03662 & $8.044 \mathrm{E}-6$ & $8.281 \mathrm{E}-6$ \\
1.819 & 1.819 & 3.5267427 & 0.02108 & 0.02278 & 0.03484 & 0.03664 & $8.051 \mathrm{E}-6$ & $8.288 \mathrm{E}-6$ \\
1.82 & 1.82 & 3.5267433 & 0.0211 & 0.0228 & 0.03486 & 0.03666 & $8.058 \mathrm{E}-6$ & $8.296 \mathrm{E}-6$ \\
1.821 & 1.821 & 3.5267431 & 0.02112 & 0.02282 & 0.03488 & 0.03668 & $8.066 \mathrm{E}-6$ & $8.303 \mathrm{E}-6$ \\
1.822 & 1.822 & 3.5267411 & 0.02115 & 0.02285 & 0.03491 & 0.03671 & $8.073 \mathrm{E}-6$ & $8.311 \mathrm{E}-6$ \\
$\vdots$ & $\vdots$ & $\vdots$ & $\vdots$ & $\vdots$ & $\vdots$ & $\vdots$ & $\vdots$ & $\vdots$ \\
2.413 & 2.413 & 3.417046 & 0.03589 & 0.05178 & 0.05291 & 0.06968 & $1.198 \mathrm{E}-5$ & $1.346 \mathrm{E}-5$ \\
\hline \multicolumn{7}{c}{ TABLE 1 } \\
\end{tabular}

Therefore, we find that $\lambda_{h}\left(\mu_{j}\right)+\varepsilon_{j}<\lambda_{h}\left(\mu_{l}\right)-\varepsilon_{l}$. However, this is a contradiction of the fact that $I_{j} \cap I_{l} \neq \phi$.

Lemma 4 does not make sure the uniqueness of turning points, but we can conclude the existence of ones without any special computations.

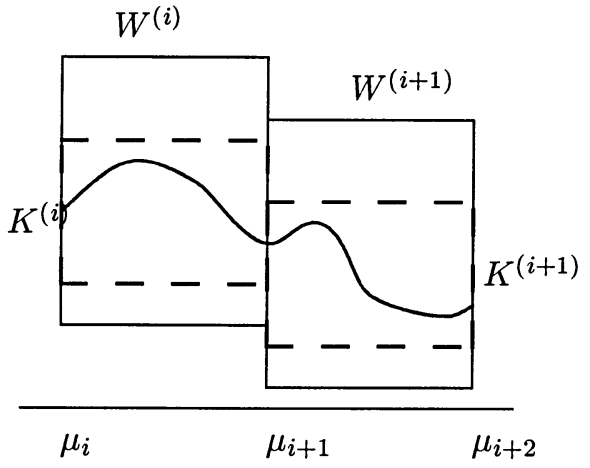

FIG. 1.

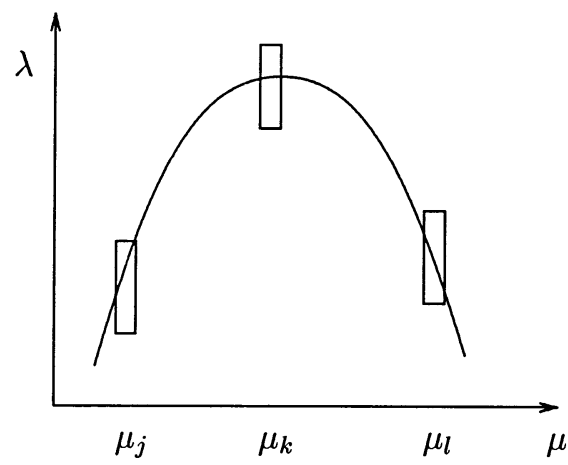

FIG. 2 .

6. Numerical Examples. We now provide a numerical example for continua computations in three dimensional case $n=3$ (Table 1). Here we use $W_{h}:=\max _{i=0, \cdots, M-1} \widetilde{W}_{i}, K_{h}:=\max _{i=0, \cdots, M-1}\left(Y_{i}+Z_{i}\right)$, and the parameter $\varepsilon$ is fixed $\varepsilon=0.05$. In these computations, we used the interval library PROFIL [7], which supports the interval linear system solvers proposed by Rump [12].

Here, $\widetilde{W}_{M}$ stands for the bound $\left|\lambda-\lambda_{h}\right|$. Choosing $\mu_{j}=1.32, \mu_{k}=1.82$ and $\mu_{l}=2.413$, the assumptions in Lemma 4 are satisfied. This means that a certain turning point exists in $\left[\mu_{j}, \mu_{l}\right]$. From Table 1 , we conclude the value 3.56341 is an upper bound of the first turning point. On the other hand, from Lemma 4 and Table 1 it seems that the value of it's lower bound is 3.3647 by computing $\lambda_{h}\left(\mu_{i}\right)-\varepsilon_{i}$ on each $e_{i}$. However, since Lemma 4 does not ensure the uniqueness of turning point, we can't guarantee that 3.3647 is an lower bound of the 'first' turning point. We carried out our numerical verification process without a bordering equation to find the lower bound of definitely first turning point. As the result, it is found that there is no turning point in $\lambda=[0,3.4]$. From this fact, Table 1 and Figure 3, it is apparent that the first turning point (the explosive point) is contained in the interval [3.4, 3.56341]. 


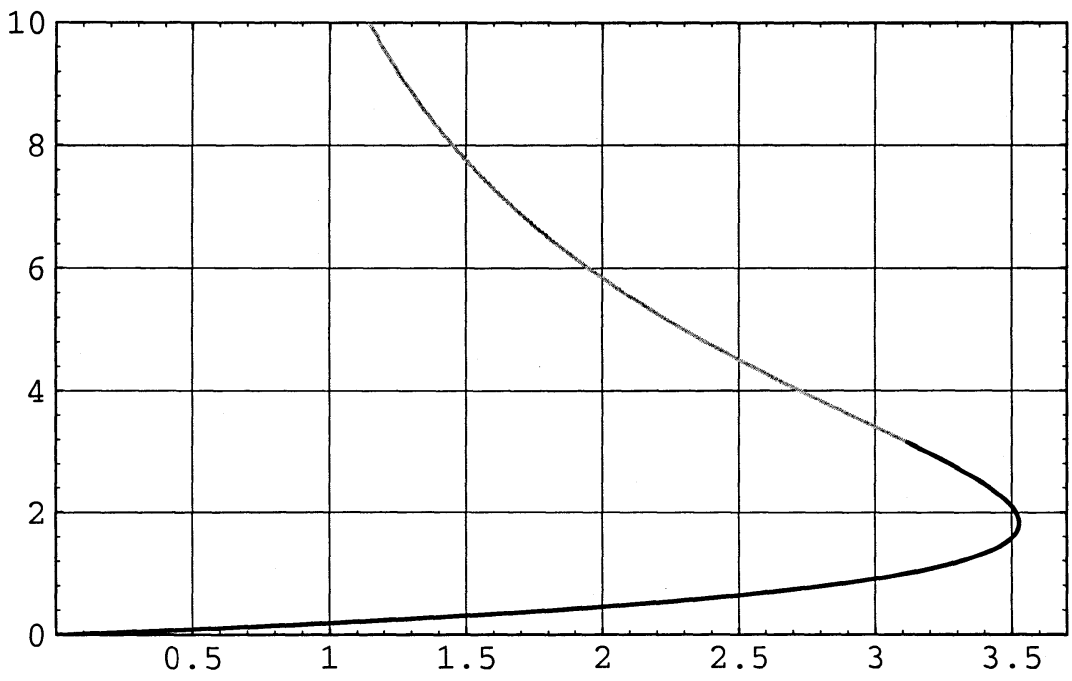

Fig. 3. Bifurcation diagram for (2.8). The value of $\|u\|_{\infty}$ corresponds to the ordinate axis, and $\lambda$ to the abscissa. Here the black portion is the numerically verified part, and the gray portion is the approximate part.

REMARK 1. In the present article, since we used an integral equation for the radially symmetric solution of the original problem, it is easily applicable to the higher dimensional case. This is the advantage of our method. On the other hand, other methods, e.g., [10] seem to be difficult to apply directly, even if the integral form is used such as (2.2), because the method requires to estimate the eigenvalue with smallest absolute value of the inverse of the linearized operator for nonlinear problems.

\section{REFERENCES}

[1] G. Alefeld And J. Herzberger, Introduction to Interval Computations, Academic Press, New York, 1983.

[2] J. Bebernes AND D. Eberly, Mathematical Problems from Combustion Theory, SpringerVerlag, 1989.

[3] E. N. DANCER, On the structure of solutions of an equation in Catalysis theory when a parameter is large, J. Diff. Equations, 37(1980), pp. 404-437.

[4] D. A. Frank-Kamenetskit, Diffusion and Heat Transfer in Chemical Kinetics, 2nd edition, Plenum Press, 1969.

[5] D. Gilbarg and N. S. Trudinger, Elliptic Partial Differential Equations of Second Order, 2nd edition, Springer, 1983.

[6] H. B. Keller, Lectures on Numerical Methods in Bifurcation Problems, Springer, 1987.

[7] O. KNÜPPEL, PROFIL/BIAS - A fast interval library, Computing, 53 (1994), pp. 277-288.

[8] M. T. NAKAO AND N. YAMAмоTO, Numerical verification of solutions for nonlinear elliptic problems using an $L^{\infty}$ residual method, J. Math. Anal. Appl., 217 (1998), pp. 246-262.

[9] J. R. PARKS, Criticality criteria for various configurations of a self-heating chemical as functions of activation energy and temperature of assembly, J. Chem. Phys., 34 (1961), pp. 46-50.

[10] M. PLUM, Existence and enclosure results for continua of solutions of parameter-dependent nonlinear boundary value problems, Journal of Computational and Applied Mathematics, 60 (1995), pp. 187-200.

[11] W. C. Rheinboldt, Numerical Analysis of Parameterized Nonlinear Equations, Wiley, 1986.

[12] S. M. Rump, Solving algebraic problems with high accuracy, A new approach to scientific computation, U. Kulisch and W. L. Miranker, ed., Academic Press, 1983. 
[13] R. SEYdel, Practical Bifurcation and Stability Analysis, 2nd edition, Springer, 1991.

[14] M. H. Schultz, Spline Analysis, Prentice-Hall, 1973.

[15] K. Taira and K. Umezu, Positive solutions of sublinear elliptic boundary value problems, Nonlinear Analysis, 29 (1997), pp. 761-771.

[16] K. K. TAM, Construction of upper and lower solutions for a problem in combustion theory, J. Math. Anal. Appl., 69 (1979), pp. 131-145.

[17] T. TSUCHIYA AND I. BABUŠKA, A priori error estimates of finite element solutions of parameterized strongly nonlinear boundary value problems, Journal of Computational and Applied Mathematics, 79 (1997), pp. 41-66.

[18] T. TsUCHIYA AND M. T. NAKAO, Numerical verification of solutions of parameterized nonlinear boundary value problems with turning points, Japan J. Indust. Appl. Math., 14 (1997), pp. $357-372$.

[19] N. YAMAMOTO, A numerical verification method for solutions of boundary value problems with local uniqueness by Banach's fixed point theorem, SIAM J. Numer. Anal., 35 (1998), pp. 2004-2013.

[20] E. ZEIDLER, Nonlinear Functional Analysis and its Applications I, Springer-Verlag, New York, 1986. 